\title{
Unconventional scaling and significant enhancement of the spin Seebeck effect in multilayers
}

\author{
R. Ramos, ${ }^{1,2,3,{ }^{*}}$ T. Kikkawa, ${ }^{2,4}$ M. H. Aguirre, ${ }^{1,5,6}$ I. Lucas, ${ }^{1,7}$ A. Anadón,,${ }^{1,5}$ T. Oyake, ${ }^{8}$ K. Uchida, ${ }^{4,9}$ H. Adachi, ${ }^{3,10}$ \\ J. Shiomi, ${ }^{8}$ P. A. Algarabel, ${ }^{5,11}$ L. Morellón, ${ }^{1,5}$ S. Maekawa, ${ }^{3,10}$ E. Saitoh, $, 3,4,10$ and M. R. Ibarra ${ }^{1,5,6}$ \\ ${ }^{1}$ Instituto de Nanociencia de Aragón, Universidad de Zaragoza, E-50018 Zaragoza, Spain \\ ${ }^{2}$ WPI Advanced Institute for Materials Research, Tohoku University, Sendai 980-8577, Japan \\ ${ }^{3}$ Spin Quantum Rectification Project, ERATO, Japan Science and Technology Agency, Sendai 980-8577, Japan \\ ${ }^{4}$ Institute for Materials Research, Tohoku University, Sendai 980-8577, Japan \\ ${ }^{5}$ Departamento de Física de la Materia Condensada, Universidad de Zaragoza, E-50009 Zaragoza, Spain \\ ${ }^{6}$ Laboratorio de Microscopías Avanzadas, Universidad de Zaragoza, E-50018 Zaragoza, Spain \\ ${ }^{7}$ Fundación ARAID, E-50018 Zaragoza, Spain \\ ${ }^{8}$ Department of Mechanical Engineering, The University of Tokyo, Tokyo 113-8656, Japan \\ ${ }^{9}$ PRESTO, Japan Science and Technology Agency, Saitama 332-0012, Japan \\ ${ }^{10}$ Advanced Science Research Center, Japan Atomic Energy Agency, Tokai 319-1195, Japan \\ ${ }^{11}$ Instituto de Ciencia de Materiales de Aragón, Universidad de Zaragoza and Consejo Superior de Investigaciones Científicas, \\ E-50009 Zaragoza, Spain
}

(Received 4 June 2015; published 11 December 2015)

\begin{abstract}
Spin Seebeck effects (SSEs) have been investigated in highly crystalline magnetic multilayer $\left[\mathrm{Fe}_{3} \mathrm{O}_{4} / \mathrm{Pt}\right]_{n}$ films. Voltage as well as power generated by the SSE were found to be significantly enhanced with increasing the number of layers $n$. This voltage enhancement defies the simple understanding of the SSE and suggests that spin current flowing between the magnetic layers in the thickness direction plays an important role in multilayer SSE systems and the observed voltage enhancement.
\end{abstract}

DOI: 10.1103/PhysRevB.92.220407

PACS number(s): 72.15.Jf, 72.25.-b, 85.75.-d

The generation and manipulation of spin currents are central aspects of spintronics. The recently discovered spin Seebeck effect (SSE) [1] is of significant importance within this field, since it enables simple and versatile generation of spin currents from heat. The SSE refers to the thermal generation of a spin current from a ferromagnetic material $(F)$ into a nonmagnetic conductor $(\mathrm{N})$ in contact with the ferromagnet, via thermal magnetization dynamics. The thermally generated spin current $\left(J_{S}\right)$ is converted in the $\mathrm{N}$ layer into an electric voltage (SSE voltage) by means of the inverse spin Hall effect (ISHE) [2-5] as a result of the spin-orbit interaction [Fig. 1(a)].

The SSE has been experimentally established and observed in ferromagnetic materials: metals [1], semiconductors [6,7], and oxides [8-10]. Theoretical models have also been developed [11-15]. This has contributed to the rapid expansion of the field of thermospin effects [16-20], which is focused on the study of the correlation between charge, heat, and spin currents in magnetic materials. Furthermore, the observation of the SSE in magnetic insulators [8] is of great interest for potential applications as in energy harvesting [21,22], since it allows the generation of electric voltages without the thermal loss associated with mobile charge carriers, opening the possibility to explore magnetic oxides, not previously studied in thermoelectric generation. Another inherent advantage of the SSE is that it involves properties of at least two different materials that can be optimized independently. However, the SSE reported up to now is small [23]. One approach for the improvement of the observed signal is aimed at the enhancement of the spin current detection, by exploiting the spin Hall angle characteristics of the $\mathrm{N}$ layer [24], and one

*ramosr@imr.tohoku.ac.jp such example is the SSE voltage enhancement in spin Hall thermopiles [25], however, there is no power improvement associated with the voltage increase [26] due to the large internal resistance of these devices. Another possibility to increase the SSE voltage could be directed towards an increase of the generated spin current. Therefore, further understanding of the thermal spin current generation process is of utmost importance to develop fundamental concepts that can be applied to enhance the SSE.

In this Rapid Communication, we have investigated SSE in magnetic multilayers formed by the repeated growth of $n$ number of $\mathrm{F} / \mathrm{N}$ bilayers, hereafter referred to as $[\mathrm{F} / \mathrm{N}]_{n}$ [Fig. 1(b)]. This type of system provides a unique template to investigate the physics of spin current at the interfaces.

In a F/N bilayer $(n=1)$ [Fig. 1(c)], the SSE is explained as a result of a spin current $\left(J_{S}\right)$ injected from the ferromagnetic material into a nonmagnetic conductor, where it is converted into an electric voltage $\left(V_{\mathrm{BL}}\right)$ by the ISHE. The SSE is a consequence of the thermally induced nonequilibrium between magnons in $\mathrm{F}$ and conduction-electron spin in $\mathrm{N}$ at the $\mathrm{F} / \mathrm{N}$ interface.

Let us now consider a double bilayer system, $n=2$ $(\mathrm{F} / \mathrm{N} / \mathrm{F} / \mathrm{N})$. In the simplest case, the system can be treated as two independent bilayers electrically connected in parallel. For a constant thermal gradient applied in the thickness direction $(\| z)$ and assuming heat flow conservation, the injected spin current is expected to remain constant across all the $\mathrm{F} / \mathrm{N}$ interfaces of the system $\left(J_{S 1}=J_{S 1^{\prime}}=J_{S 2}\right)$ [Fig. 1(d)]. In this scenario a power increase is expected, as a consequence of the reduced resistance due to the parallel connection of two bilayers. A voltage enhancement could only be observed in the $\mathrm{N}_{1}$ layer due to spin current injection from the top and bottom surface. As for multilayer systems $(n \geqslant 3)$, the 


\section{(a) SSE in conventional bilayer}

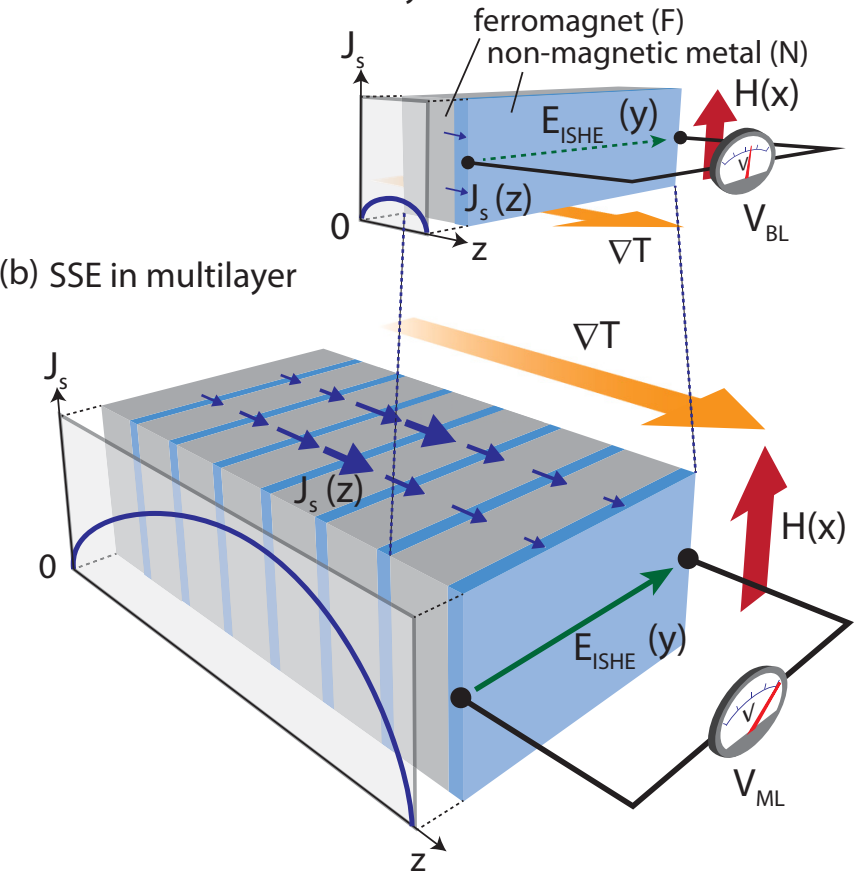

(c) Bilayer SSE:

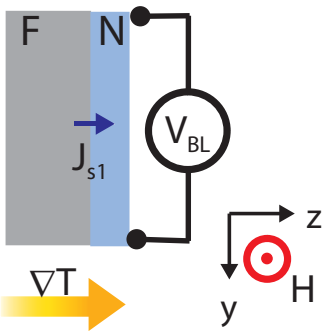

(d) Gedanken double bilayer

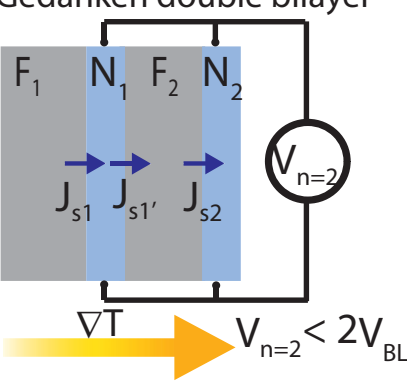

FIG. 1. (Color online) (a), (b) Schematic illustrations of (a) the $\mathrm{SSE}$ in an individual F/N bilayer and (b) the giant SSE in a magnetic multilayer formed by repetition of F/N bilayers. The theoretically predicted dependence of the spin current $\left(J_{S}\right)$ across the multilayer is also sketched [the exact calculated dependence is outlined in the Supplemental Material (SM), Sec. VI [29]]. (c) Schematic illustration of the thermally generated spin currents $\left(J_{S}\right)$ for a single $\mathrm{F} / \mathrm{N}$ bilayer. (d) Gedanken experiment of a double bilayer $(n=2)$ considering the conventional picture of SSE in F/N single bilayers.

maximum voltage that can be achieved under this picture could be obtained for a multilayer $[\mathrm{F} / \mathrm{N}]_{n}$ with very large $n$, with an estimated SSE voltage of $V \approx 2 V_{\mathrm{BL}}$. This value represents the upper limit for the expected voltage, considering the conventional SSE understanding.

Here, we show that a significantly larger SSE voltage enhancement can be attained in a multilayer system. Although previous studies have also shown an enhancement of the transversal thermoelectric voltage in $[\mathrm{F} / \mathrm{N}]_{n}$ multilayers, both the $\mathrm{F}$ and $\mathrm{N}$ layers are metallic materials and the anomalous Nernst effect (ANE) and SSE are strongly entangled [27,28]. In contrast, we have studied magnetic multilayers based on the repeated growth of magnetite/platinum bilayer thin films [Fig. 2(a)] [29]. Magnetite $\left(\mathrm{Fe}_{3} \mathrm{O}_{4}\right)$ is a magnetic oxide, extensively studied in spintronics $[30,31]$ due to its fascinating (a)

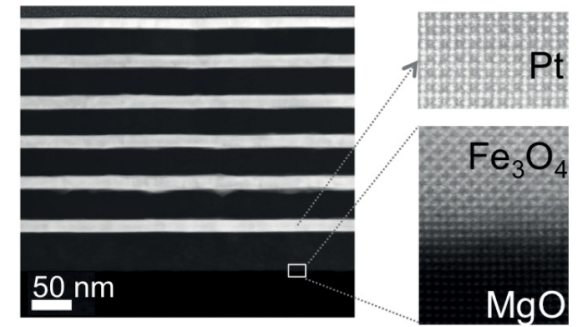

(b)

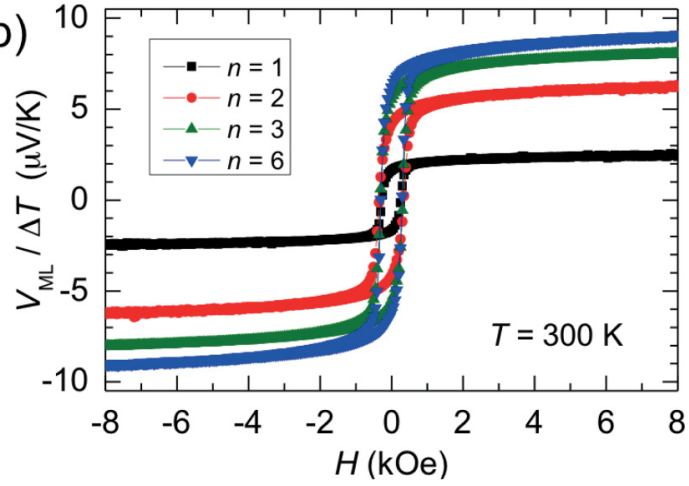

FIG. 2. (Color online) (a) Scanning transmission electron microscopy image of the cross section of a $\left[\mathrm{Fe}_{3} \mathrm{O}_{4}(34) / \mathrm{Pt}(17)\right]_{6}$ sample (thickness in $\mathrm{nm}$ ). Details of the $\mathrm{Pt}$ layer and $\mathrm{MgO} / \mathrm{Fe}_{3} \mathrm{O}_{4}$ interface are shown to demonstrate the high crystalline quality of the multilayers. (b) $H$ dependence of the SSE in $\left[\mathrm{Fe}_{3} \mathrm{O}_{4}(34) / \mathrm{Pt}(17)\right]_{n}$ for different numbers of bilayers $n$.

magnetic properties [32-36], and platinum ( $\mathrm{Pt}$ ), a nonmagnetic metal with strong spin-orbit interactions, used as the spin current detector. In this system the SSE is the dominant contribution, despite $\mathrm{Fe}_{3} \mathrm{O}_{4}$ being electrically conductive at room temperature. This is because the electrical resistivity of $\mathrm{Fe}_{3} \mathrm{O}_{4}$ is much greater than that of $\mathrm{Pt}$, where the ANE contribution is strongly reduced due to short-circuit effects [9].

We measured the contribution of the ANE of the magnetite layer, which accounts for less than $1 \%$ of the measured voltage, implying that the observed effect is dominated by the SSE of the $\mathrm{Fe}_{3} \mathrm{O}_{4} / \mathrm{Pt}$ bilayer, in good agreement with our previous study [9]. This is also supported by measurements varying the directions of the applied magnetic field and thermal gradient with respect to the $\mathrm{Fe}_{3} \mathrm{O}_{4} / \mathrm{Pt}$ interface [37], which show the absence of both ANE in a $\mathrm{Fe}_{3} \mathrm{O}_{4} / \mathrm{Pt}$ bilayer and ANE due to a transport magnetic proximity effect in Pt (SM, Sec. III [29]). Furthermore, even if there was a transport magnetic proximity effect in Pt, the voltage signal from such a proximity effect would not scale with the number of layers because each layer is connected in parallel electrically. The upper limit of the transport magnetic proximity effect in the present multilayer system, which is inferred from the systematic study of the effect in a $\mathrm{Y}_{3} \mathrm{Fe}_{5} \mathrm{O}_{12} / \mathrm{Pt}$ system [37], is far below the observed SSE enhancement. This is also supported by a recent report showing that the magnetic proximity effect in a F/N magnetic multilayer is negligible [27].

The SSE measurements were performed by applying an external magnetic field in the $x$ direction while a temperature difference $\Delta T$ between the bottom and top surfaces of the sample is maintained, parallel to the $z$ direction [Fig. 1(b)]. The SSE voltage generated by the thermally induced $E_{\text {ISHE }}$ is measured by contacting the Pt top layer along the $y$ 
direction [29]. The dimensions of the samples are $L_{x}=2 \mathrm{~mm}$, $L_{y}=7 \mathrm{~mm}$, and $L_{z}=0.5 \mathrm{~mm}$.

Figure 2(b) shows the $H$ dependence of the measured SSE voltage $\left(V_{\mathrm{ML}}\right)$ in the multilayer system, normalized by $\Delta T$, for different numbers of $\mathrm{Fe}_{3} \mathrm{O}_{4}(34) / \mathrm{Pt}(17)$ bilayers $n$. The data show that the SSE voltage scales up as $n$ is increased. This enhancement cannot be explained by the dependence of the SSE on the thickness of ferromagnetic material, as evidenced from the SSE voltage measured for a $\mathrm{Fe}_{3} \mathrm{O}_{4}(278) / \mathrm{Pt}(14)$ sample, which is much lower than the value of the multilayer with $n=6$ (SM, Sec. IV [29]). This shows that the SSE increase is intrinsically related to the multilayered structure.

The observed enhancement is surprising, since it defies the aforementioned simple understanding of the SSE. The measured voltage enhancement is significantly larger than what was previously expected [Fig. 1(d)]. Therefore, another mechanism should be introduced for the SSE in multilayers. One scenario that should be discarded is the possible reduction of the thermal conductivity with an increasing number of layers as a consequence of the increased phonon scattering across multiple interfaces [38]. Thermoreflectance measurements show a nearly constant value of the thermal conductivity upon increasing the number of bilayers. The average measured value was $2.9 \pm 0.2 \mathrm{~W} / \mathrm{m} \mathrm{K}$, independent of the number of bilayers (SM, Sec. V [29]), thus the reduction of the thermal conductivity in the multilayer system is not the reason behind our observations.

In order to understand the origin of the observed phenomenon, we performed test experiments to investigate how the SSE is influenced by varying the nature and thickness of the nonmagnetic interlayers. First, we measured a multilayer system in which the inner Pt interlayers of the sample were replaced with $\mathrm{MgO}$, leaving only the topmost $\mathrm{Pt}$ layer. In this structure, heat transport across the multilayer thickness is maintained (due to the high thermal conductivity of $\mathrm{MgO}$ ), while the electron/spin transport across $\mathrm{MgO}$ is suppressed. Figure 3(a) shows the comparative results obtained for $\mathrm{Fe}_{3} \mathrm{O}_{4}(34) / \mathrm{Pt}(17), \quad\left[\mathrm{Fe}_{3} \mathrm{O}_{4}(34) / \mathrm{Pt}(17)\right]_{3}$, and $\left[\mathrm{Fe}_{3} \mathrm{O}_{4}(34) / \mathrm{MgO}(8)\right]_{2} / \mathrm{Fe}_{3} \mathrm{O}_{4}(34) / \mathrm{Pt}(17)$. It is interesting to observe that, despite having similar thermal conductivities, the multilayer system with $\mathrm{MgO}$ interlayers shows a strong reduction of the SSE signal as a consequence of the absence of spin current propagation through the $\mathrm{MgO}$ interlayers. The measured SSE value is comparable to the one obtained for a single $\mathrm{Fe}_{3} \mathrm{O}_{4} / \mathrm{Pt}$ bilayer. This result is a strong indication that the observed giant SSE is a purely spin current effect, in which the existence of multiple $\mathrm{Fe}_{3} \mathrm{O}_{4} / \mathrm{Pt}$ interfaces is a relevant factor.

Second, to further study the role of the $\mathrm{N}$ interlayers in the SSE voltage enhancement, we performed measurements of a $\left[\mathrm{Fe}_{3} \mathrm{O}_{4}(23) / \mathrm{Pt}(7)\right]_{6}$ multilayer, where the Pt thickness is reduced to a value just above the reported spin diffusion length of Pt [39]. This sample presents a giant SSE $(\sim 25 \mu \mathrm{V} / \mathrm{K})$, with a substantial increase with respect to the one observed for a single bilayer [Fig. 3(b)]. This result shows the direct relation between the length scale for spin transport in the nonmagnetic metal and the observed voltage enhancement, clearly indicating the relevance of the spin current propagation across the Pt interlayers in the observed phenomenon.
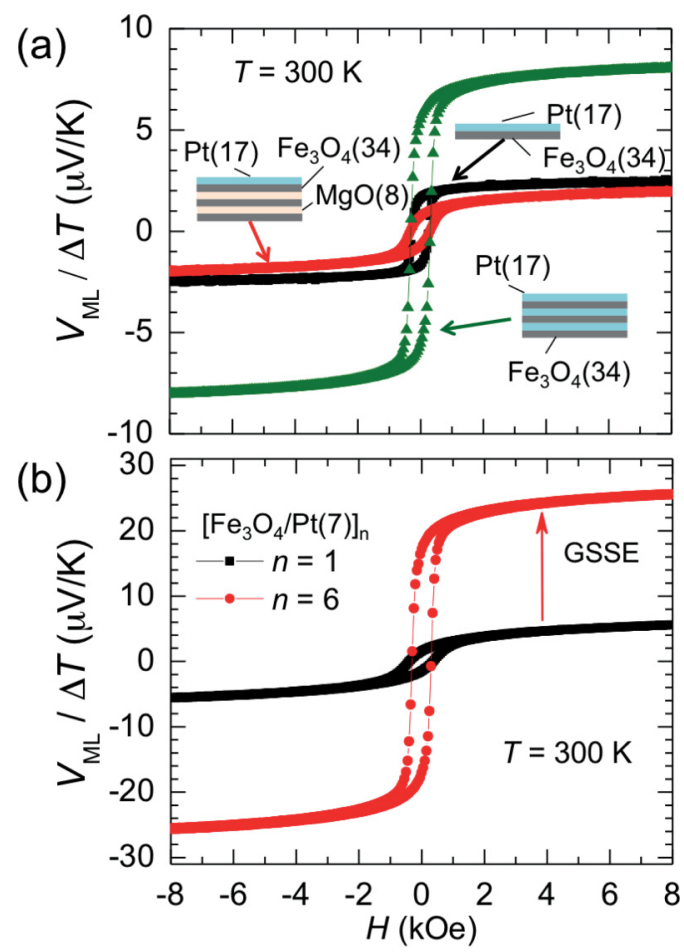

FIG. 3. (Color online) (a) Effect of Pt replacement by $\mathrm{MgO}$ interlayers (electrical insulator and thermal conductor): the data show the comparison of the SSE between $\mathrm{Fe}_{3} \mathrm{O}_{4} / \mathrm{Pt},\left[\mathrm{Fe}_{3} \mathrm{O}_{4} / \mathrm{Pt}\right]_{3}$ and $\left[\mathrm{Fe}_{3} \mathrm{O}_{4} / \mathrm{MgO}_{2} / \mathrm{Fe}_{3} \mathrm{O}_{4} / \mathrm{Pt}\right.$. (b) $\mathrm{SSE}$ of a $\mathrm{Fe}_{3} \mathrm{O}_{4}(23) / \mathrm{Pt}(7)$ bilayer compared with the giant SSE (GSSE) observed in a $\left[\mathrm{Fe}_{3} \mathrm{O}_{4}(23) / \mathrm{Pt}(7)\right]_{6}$ multilayer.

Before discussing our result, we need to consider the fact that the lateral dimensions of our samples are about six orders of magnitude larger than the thickness of the F/N bilayers, therefore, the out-of-plane resistance is negligibly small compared to the in-plane resistance (also supported by resistivity measurements in the multilayers-SM, Sec. VII [29]). This implies that, even if we are placing the electrical contacts on the topmost Pt layer, we can sense the SSE voltage in all the Pt layers simultaneously, thus approximately observing an averaged SSE voltage. Therefore, to explain the unexpected SSE voltage enhancement, an increase of the overall spin current in the multilayer system must be considered. It is also worth mentioning that, as a consequence of the parallel connection of the Pt layers of the multilayer structure, a simultaneous increase of the voltage and the sample conductivity $(\sigma)$ occurs. This translates into an enhancement of the power factor (proportional to $\sigma V_{\mathrm{ML}}^{2}$ ) of the system [28], with an increase of more than one order of magnitude for the $\left[\mathrm{Fe}_{3} \mathrm{O}_{4} / \mathrm{Pt}\right]_{6}$ multilayer, and we believe this factor can be further enhanced by increasing $n$.

In the following, we will attempt to explain the origin of the measured voltage enhancement. The simplified picture of the SSE, described previously [Fig. 1(d)], fails to interpret the magnitude of the observed increase in SSE voltage, predicting a maximum value of $2 V_{\mathrm{BL}}$ for a multilayer $[\mathrm{F} / \mathrm{N}]_{n}$ with very large $n$. Then, if the spatial distribution and the boundary conditions for spin current propagation across the thickness direction are considered, the magnitude of the thermally 

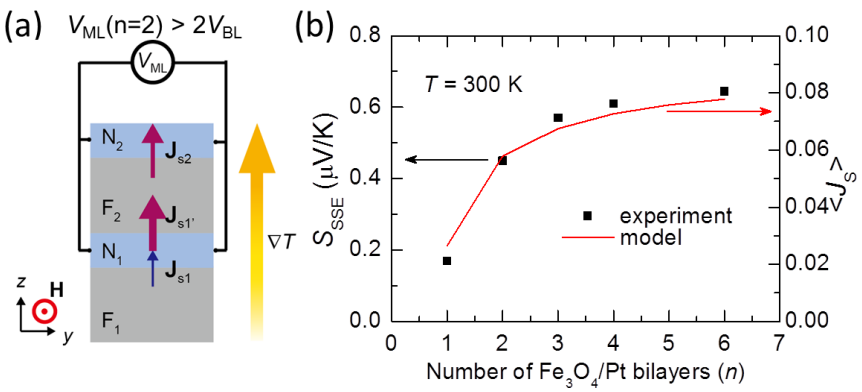

FIG. 4. (Color online) (a) Schematic illustration of the spin current enhancement in a double bilayer $(n=2)$ when considering the theoretical model developed for SSE in magnetic multilayers. (b) Comparison of the $n$ dependence between the measured SSE coefficient $\left[S_{\mathrm{SSE}}=\left(V_{\mathrm{ML}}^{\mathrm{sat}} / \Delta T\right)\left(L_{z} / L_{y}\right)\right]$ and the theoretical model prediction of the normalized average spin current $\left(\left\langle J_{S}\right\rangle\right)$ for a multilayer structure with $\left[\mathrm{Fe}_{3} \mathrm{O}_{4}(34) / \mathrm{Pt}(17)\right]_{n}$.

generated spin current in the multilayer system is expected to increase [Fig. 4(a)]. This results in an enhancement of the SSE voltage, with a magnitude significantly larger than that expected from the conventional SSE picture $\left(V_{\mathrm{ML}}>2 V_{\mathrm{BL}}\right)$.

Let us now introduce the details of our model to qualitatively explain the physics behind the presented results. This model considers a magnon spin current in the $\mathrm{F}$ layer $[15,40]$ and a conduction-electron spin current in the $\mathrm{N}$ layer. We impose as boundary conditions that the conductionelectron/magnon spin current should vanish at the top/bottom surface of the multilayer structure and the continuity of the magnon/conduction-electron spin currents at the $\mathrm{Fe}_{3} \mathrm{O}_{4} / \mathrm{Pt}$ interfaces [41]. Under these conditions, the spin current in the inner $\mathrm{N}$ layers of the structure remains a nonzero value and an enhancement of the spin current in the multilayer system is predicted, with a maximum value at the central interlayers of the structure, as it is schematically shown in Fig. 1(b) (SM, Sec. VI [29]).

The multilayer effect is explained by (i) the appearance of a new length $\xi$ that characterizes the overall variation of the spin current in the multilayer, and (ii) the existence of $\mathrm{N}$ interlayers that detect the largest spin current at the middle of the multilayer sample. Indeed, in the Supplemental Material [Sec. VI, Eq. (S16)], we show that the envelope curve of the spin current distribution in the multilayer [Fig. S10(a)] is characterized by a new length $\xi=\sqrt{G_{S}}\left(t_{F}+t_{N}\right) / \sqrt{2\left[\left(t_{F} / \Lambda\right)+\left(t_{N} / \lambda\right)\right]}$, where $G_{S}$ is a dimensionless spin conductance for the F/N interface, the expression of which is given below Eq. (S14) in SM. Then, the overall variation of the spin current is characterized by the envelope function, $J_{S}(z)=-L_{m} \nabla T\left[1-\frac{\cosh [(z-L / 2) / \xi]}{\cosh (L / 2 \xi)}\right]$, where $L_{m}$ is the magnon version of the Seebeck coefficient and $L=n\left(t_{F}+t_{N}\right)$ is the total thickness of the multilayer. This curve has a maximum at the middle of the multilayer sample, $z=L / 2$, and its value increases until $L$ exceeds $\xi$. Note that this maximum spin current at the middle of the sample can be converted into an electric signal via the ISHE only when $\mathrm{N}$ interlayers exist around the sample center, which is not the case for a conventional single bilayer. From the above expression, an estimate of the asymptotic value of the signal in the large $n$ limit can be obtained as follows. The multilayer SSE in the large $n$ limit is given by the voltage produced by the spin current at the center of the sample, $J_{S}(z=L / 2) \approx\left(-L_{m} \nabla T\right)$ for $L / \xi \gg 1$. For the single bilayer, on the other hand, the signal is determined by the spin current at the top layer, $J_{S}\left(z=L-t_{N}\right) \approx\left(-L_{m} \nabla T\right)\left(t_{N} / \xi\right)$ for $L / \xi \gg 1$. Therefore, the asymptotic value of the enhancement factor is estimated to be $\xi / t_{N}$.

The averaged spin Seebeck voltage in the multilayer structure $\left(V_{\mathrm{ML}}\right)$, proportional to the average of the spin current over all $\mathrm{N}$ layers $\left(\left\langle J_{S}\right\rangle\right)$ can be estimated by $V_{\mathrm{ML}} \propto \theta_{\mathrm{SH}} \rho_{\mathrm{N}}\left\langle J_{S}\right\rangle$, where $\theta_{\mathrm{SH}}$ and $\rho_{\mathrm{N}}$ are the spin Hall angle and resistivity of the $\mathrm{N}$ layer, respectively. Figure 4(b) shows a comparison of the experimental values of SSE and the model predictions for $\left\langle J_{S}\right\rangle$, and we can clearly see that the model reproduces the main features of the observed SSE in $[\mathrm{F} / \mathrm{N}]_{n}$ multilayers, strongly suggesting that the increase of the spin current is responsible for the observed enhancement of the spin Seebeck voltage upon increasing the number of bilayers. This interpretation is also supported by test experiments performed in $\mathrm{Fe}_{3} \mathrm{O}_{4} / \mathrm{Pt} / \mathrm{Fe}_{3} \mathrm{O}_{4}$ trilayers, where an intermediate SSE voltage between $n=1$ and $n=2$ is measured, in agreement with our model (SM, Sec. VIII [29]).

In summary, we measured SSE in highly crystalline multilayer $\left[\mathrm{Fe}_{3} \mathrm{O}_{4} / \mathrm{Pt}\right]_{n}$ films, and found that the SSE voltage significantly enhances with increasing $n$. This effect suggests an overall increase of the out-of-plane spin current in the multilayer, which translates into an enhancement of one order of magnitude of the measured SSE.

The microscopy works were conducted in the Laboratorio de Microscopias Avanzadas at Instituto de Nanociencia de Aragon, Universidad de Zaragoza. This work was supported by the Spanish Ministry of Science (projects PRI-PIBJP2011-0794, MAT2011-27553-C02, FEDER funding), Aragón Regional Government (E26), Thermo-spintronic Marie-Curie CIG (Grant Agreement No. 304043), JST-CREST "Creation of Nanosystems with Novel Functions through Process Integration," JST-PRESTO "Phase Interfaces for Highly Efficient Energy Utilization," Grant-in-Aid for Scientific Research on Innovative Areas "Nano Spin Conversion Science" (26103005), Grant-in-Aid for Challenging Exploratory Research (26600067), Grant-in-Aid for Scientific Research (A) (15H02012) from MEXT, Japan, and NEC Corporation. T.K. and T.O. are supported by JSPS through a research fellowship for young scientists (15J08026 for T.K. and 14J09110 for T.O.).
[1] K. Uchida, S. Takahashi, K. Harii, J. Ieda, W. Koshibae, K. Ando, S. Maekawa, and E. Saitoh, Nature (London) 455, 778 (2008)

[2] E. Saitoh, M. Ueda, H. Miyajima, and G. Tatara, Appl. Phys. Lett. 88, 182509 (2006).

[3] S. O. Valenzuela and M. Tinkham, Nature (London) 442, 176 (2006).
[4] T. Kimura, Y. Otani, T. Sato, S. Takahashi, and S. Maekawa, Phys. Rev. Lett. 98, 156601 (2007).

[5] M. V. Costache, M. Sladkov, S. M. Watts, C. H. van Der Wal, and B. J. van Wees, Phys. Rev. Lett. 97, 216603 (2006).

[6] C. M. Jaworski, J. Yang, S. Mack, D. D. Awschalom, J. P. Heremans, and R. C. Myers, Nat. Mater. 9, 898 (2010). 
[7] C. M. Jaworski, J. Yang, S. Mack, D. D. Awschalom, R. C. Myers, and J. P. Heremans, Phys. Rev. Lett. 106, 186601 (2011).

[8] K. Uchida, J. Xiao, H. Adachi, J. Ohe, S. Takahashi, J. Ieda, T. Ota, Y. Kajiwara, H. Umezawa, H. Kawai et al., Nat. Mater. 9, 894 (2010)

[9] R. Ramos, T. Kikkawa, K. Uchida, H. Adachi, I. Lucas, M. H. Aguirre, P. Algarabel, L. Morellón, S. Maekawa, E. Saitoh et al., Appl. Phys. Lett. 102, 072413 (2013).

[10] T. Niizeki, T. Kikkawa, K. Uchida, M. Oka, K. Z. Suzuki, H. Yanagihara, E. Kita, and E. Saitoh, AIP Adv. 5, 053603 (2015).

[11] J. Xiao, G. E. W. Bauer, K. Uchida, E. Saitoh, and S. Maekawa, Phys. Rev. B 81, 214418 (2010).

[12] H. Adachi, J.-I. Ohe, S. Takahashi, and S. Maekawa, Phys. Rev. B 83, 094410 (2011).

[13] H. Adachi, K. Uchida, E. Saitoh, and S. Maekawa, Rep. Prog. Phys. 76, 036501 (2013).

[14] S. Hoffman, K. Sato, and Y. Tserkovnyak, Phys. Rev. B 88, 064408 (2013).

[15] S. M. Rezende, R. L. Rodríguez-Suárez, R. O. Cunha, A. R. Rodrigues, F. L. A. Machado, G. A. Fonseca Guerra, J. C. Lopez Ortiz, and A. Azevedo, Phys. Rev. B 89, 014416 (2014).

[16] G. E. W. Bauer, E. Saitoh, and B. J. van Wees, Nat. Mater. 11, 391 (2012).

[17] S. R. Boona, R. C. Myers, and J. P. Heremans, Energy Environ. Sci. 7, 885 (2014)

[18] J. Flipse, F. L. Bakker, A. Slachter, F. K. Dejene, and B. J. van Wees, Nat. Nanotechnol. 7, 166 (2012).

[19] A. Slachter, F. L. Bakker, J.-P. Adam, and B. J. van Wees, Nat. Phys. 6, 879 (2010).

[20] M. V. Costache, G. Bridoux, I. Neumann, and S. O. Valenzuela, Nat. Mater. 11, 199 (2012).

[21] F. J. DiSalvo, Science 285, 703 (1999).

[22] A. B. Cahaya, O. A. Tretiakov, and G. E. W. Bauer, IEEE Trans. Magn. 51, 0800414 (2015).

[23] T. Kikkawa, K. Uchida, Y. Shiomi, Z. Qiu, D. Hou, D. Tian, H. Nakayama, X.-F. Jin, and E. Saitoh, Phys. Rev. Lett. 110, 067207 (2013).
[24] L. Liu, C.-F. Pai, Y. Li, H. W. Tseng, D. C. Ralph, and R. A. Buhrman, Science 336, 555 (2012).

[25] K. Uchida, T. Nonaka, T. Yoshino, T. Kikkawa, D. Kikuchi, and E. Saitoh, Appl. Phys. Express 5, 093001 (2012).

[26] K. Uchida, M. Ishida, T. Kikkawa, A. Kirihara, T. Murakami, and E. Saitoh, J. Phys.: Condens. Matter 26, 343202 (2014).

[27] K. Uchida, T. Kikkawa, T. Seki, T. Oyake, J. Shiomi, Z. Qiu, K. Takanashi, and E. Saitoh, Phys. Rev. B 92, 094414 (2015).

[28] K.-D. Lee, D.-J. Kim, H. Yeon Lee, S.-H. Kim, J.-H. Lee, K.-M Lee, J.-R. Jeong, K.-S. Lee, H.-S. Song, J.-W. Sohn et al., Sci. Rep. 5, 10249 (2015).

[29] See Supplemental Material at http://link.aps.org/supplemental/ 10.1103/PhysRevB.92.220407 for details on experimental methods, theory, and additional data and analysis.

[30] S. A. Wolf, D. D. Awschalom, R. A. Buhrman, J. M. Daughton, S. von Molnár, M. L. Roukes, A. Y. Chtchelkanova, and D. M. Treger, Science 294, 1488 (2001).

[31] I. Žutić, J. Fabian, and S. Das Sarma, Rev. Mod. Phys. 76, 323 (2004).

[32] Y. S. Dedkov, U. Rüdiger, and G. Güntherodt, Phys. Rev. B 65 , 064417 (2002).

[33] F. Walz, J. Phys.: Condens. Matter 14, R285 (2002).

[34] R. Ramos, S. K. Arora, and I. V. Shvets, Phys. Rev. B 78, 214402 (2008).

[35] H.-C. Wu, M. Abid, B. S. Chun, R. Ramos, O. N. Mryasov, and I. V. Shvets, Nano Lett. 10, 1132 (2010).

[36] R. Ramos, M. H. Aguirre, A. Anadón, J. Blasco, I. Lucas, K. Uchida, P. A. Algarabel, L. Morellón, E. Saitoh, and M. R. Ibarra, Phys. Rev. B 90, 054422 (2014).

[37] T. Kikkawa, K. Uchida, S. Daimon, Y. Shiomi, H. Adachi, Z Qiu, D. Hou, X.-F. Jin, S. Maekawa, and E. Saitoh, Phys. Rev. B 88, 214403 (2013).

[38] M. V. Simkin and G. D. Mahan, Phys. Rev. Lett. 84, 927 (2000).

[39] V. Castel, N. Vlietstra, J. Ben Youssef, and B. J. van Wees, Appl. Phys. Lett. 101, 132414 (2012).

[40] Y. Kajiwara, K. Harii, S. Takahashi, J. Ohe, K. Uchida, M. Mizuguchi, H. Umezawa, H. Kawai, K. Ando, K. Takanashi et al., Nature (London) 464, 262 (2010).

[41] S. S.-L. Zhang and S. Zhang, Phys. Rev. B 86, 214424 (2012). 